\title{
Plasmon electron energy-loss spectroscopy and in-situ cooling experiments of graphene liquid cells
}

\author{
Lopa Bhatt ${ }^{1}$, Jinglong $\mathrm{Guo}^{2}$ and Robert $\mathrm{Klie}^{3}$ \\ ${ }^{1}$ University of Illinois at Chicago, United States, ${ }^{2}$ University of Illinois at Chicago, Cupertino, California, \\ United States, ${ }^{3}$ University of Illinois at Chicago, Chicago, Illinois, United States
}

Water is the most important chemical compound on earth for living organisms. The properties of bulk water have been studied intensively for several decades. In technologically relevant scenarios, water is often confined in small cavities which leads to emergence of novel phases that are not observed in bulk water under the same thermodynamic conditions. Yet, only recently have the structure, properties and phase transitions effects of confined water and its related phases been the subject of more thorough investigations,[1] primarily due to the limitation in spatial resolution or chemical sensitivity/selectivity of the experimental approach. One of these experimental methods is transmission electron microscopy (TEM), a technique that provides unprecedented spatial resolution in crystalline material, but, until the last few years, has not been used to study liquids. This is mostly due to instability of aqueous solution in high vacuum conditions of the TEM column and inability to measure in-situ temperature while preserving atomic-resolution.

In recent years, liquid cells (LC) have been developed as one way to contain liquid in a TEM column and enable the study of solutions.[2] In LC, an aqueous solution is encapsulated between 2D film membranes such as silicon nitride and graphene, which shield the liquid from the vacuum environment in the TEM column as shown in Figure 1.[3] Using graphene liquid cells (GLC) appears to alleviate some of the electron beam induced radiolysis effects and new approaches, such as aloof spectroscopy now enables virtually damage free chemical analysis.

To accurately measure the temperature of the aqueous sample under electron beam while being able to get atomic resolution images has been a challenge in field of electron microscopy. While several methods, including diffraction and electron-energy gain spectroscopy have been developed over the years, plasmonbased thermometry is one of the only approaches that combines high spatial resolution and accuracy of temperature of material under investigation. In plasmon-based thermometry, the shift of plasmon energy as a function of temperature is measured which provides a direct means of calibrating the temperature. This technique has been previously used to calibrate temperature of various 2D materials but has never been applied to water largely due to the significant challenges in working with liquid sample in high vacuum.[4]

The focus of the project presented here is to utilize aberration-corrected scanning transmission electron microscopy combined with electron energy-loss spectroscopy (EELS) to map the thermal expansion coefficient as a function of in-situ temperature of nanometer thick water layer confined between 2D materials, such as graphene. Primary data of the plasmon energy shift as a function of $\mathrm{H} 2 \mathrm{O}$ temperature using single layer GLC are shown in Figure 2. It can be seen that the plasmon peak shift, which can be directly associated with the thermal expansion coefficient $(\alpha)$ [5] tracks the calculated values of $\alpha$ very closely. This shows that using plasmonic thermometry of liquid within a TEM, it is possible to directly measure the temperature of aqueous solution during in-situ experiments. Using this technique, we will further study the phase diagram of ice in STEM. [6] 
1.

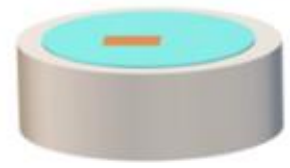

Graphene on copper substrate in etchant solution

4.

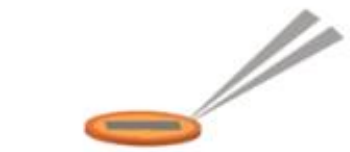

Transferring Graphene film from deionized water to TEM grid
2.

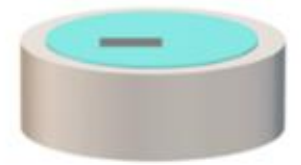

Monolayer of Graphene floating on the etchant solution

5.

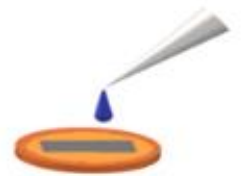

Depositing solution one wants to examine onto Graphene coated grid
3.

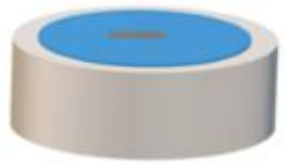

Transfer of Graphene film from etchant solution to deionized water

6.

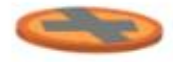

Transferring second layer of Graphene onto the grid

Figure 1. Procedure of fabricating Graphene Liquid Cell

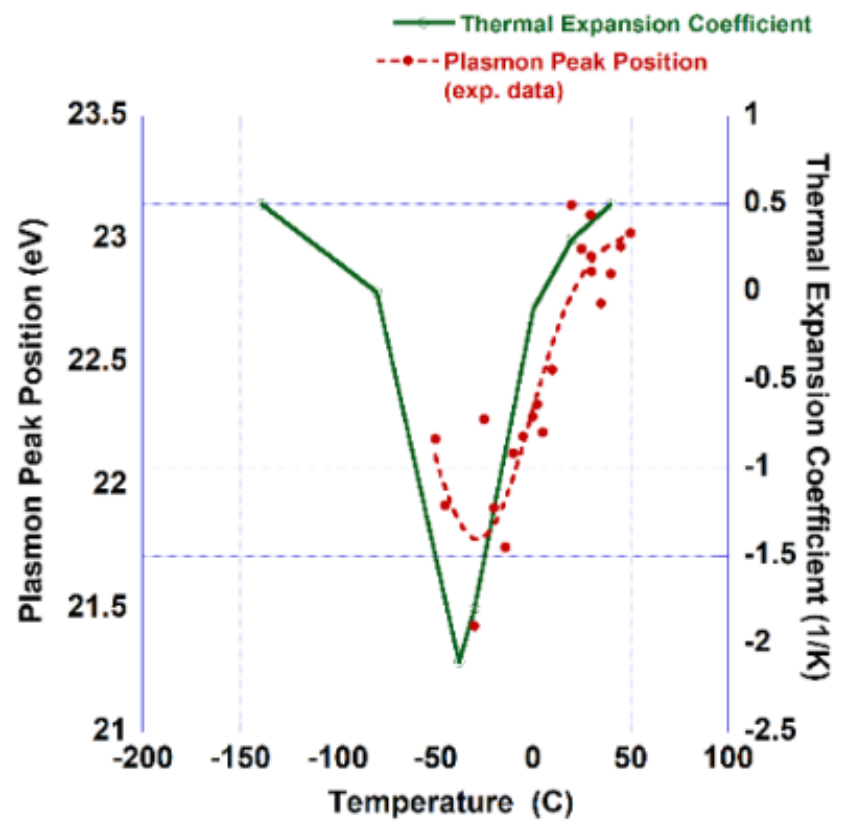

Figure 2. Graph of thermal expiation coefficient calculated using DFT (green curve) and plasmon peak positions measured using GLC (red dots) as a function of temperature

\section{References}

1. "Water in Carbon Nanotubes: The Peculiar Hydrogen Bond Network Revealed by Infrared Spectroscopy," Bernardina, S.D et al., Journal of the American Chemical Society, 138(33), (2016)

2. "Electron microscopy of specimens in liquid," de Jonge, N. and F.M. Ross, Nature Nanotechnology, 6(11), 695-704 (2011) 
3. “Applications of Graphene Liquid Cell.” Bhatt, L. et al. Microscopy and Microanalysis, 26(S2), 1452-1453 (2020)

4. "Mapping Thermal Expansion Coefficients in Freestanding 2D Materials at the Nanometer Scale," Hu, X. et al., Physical Review Letters, 120(5), 055902 (2018)

5. "Frost-heaving pressure in geotechnical engineering materials during freezing process." Wang, P. and G. Zhou, International Journal of Mining Science and Technology, 2018. 28(2): p. 287-296

6. This work is supported in part by the UIC Chancellor's Undergraduate Research Award. This work made use of Electron Microscopy Services at Research Resources Center, UIC 